\title{
Enzyme and acid deconjugation of plasma sulfated metanephrines
}

\author{
Melanie Glauser ${ }^{\mathrm{a}, 1}$, Michael Metrailler ${ }^{\mathrm{a}, 1}$, Sandrine Gerber-Lemaire ${ }^{\mathrm{b}}$, Catherine Centeno ${ }^{\mathrm{a}}$, Caroline Seghezzi ${ }^{\mathrm{a}}$, \\ Marielle Dunand ${ }^{\mathrm{a}}$, Karim Abid ${ }^{\mathrm{a}}$, Adeline Herren ${ }^{\mathrm{a}}$, Eric Grouzmann ${ }^{\mathrm{c}, *}$ \\ a Centre Hospitalier Universitaire Vaudois, Service de Biomédecine, Hôpital Beaumont, Av de Beaumont 29, CH-1011 Lausanne, Switzerland \\ ${ }^{\mathrm{b}}$ Institute of Chemistry and Chemical Engineering, Ecole Polytechnique Fédérale de Lausanne, Batochime (Bat BCH), CH-1015 Lausanne, Switzerland \\ c Service de Biomédecine, Centre Hospitalier Universitaire Vaudois, Hôpital Nestlé 5th floor, office 5017, Av Pierre Decker, 1011 Lausanne, CH-1011 Lausanne, Switzerland
}

\section{A R T I C L E I N F O}

\section{Article history:}

Received 14 October 2013

Received in revised form 5 December 2013

Accepted 30 December 2013

Available online 10 January 2014

\section{Keywords:}

Metanephrines

Sulfatase

Desulfonation

\begin{abstract}
A B S T R A C T
Background: Total (i.e. free + sulfated) metanephrines in plasma is a biomarker for the diagnosis of pheochromocytoma/paraganglioma. Sulfated metanephrines must be completely deconjugated by perchloric acid hydrolysis or sulfatase treatment prior to analytical measurement to enable quantification by current techniques. In this report, we compare the yield and efficiency of both methods.

Methods: The deconjugation rate of synthetic sulfated metanephrines (normetanephrine (S-NMN), metanephrine (S-MN) and methoxytyramine (S-MT)) spiked in charcoal-stripped plasma was determined by boiling perchloric acid and compared to sulfatase treatment. Total plasma metanephrines (MN, NMN and MT) were also determined in patient samples by both methods.

Results: The complete deconjugation of sulfated metanephrines is achieved after 30 min incubation with $0.1 \mathrm{M}$ boiling perchloric acid or upon sulfatase treatment. Ten minutes of acid hydrolysis (gold-standard) leads to a $30 \%$ underestimation of metanephrine concentrations. The enzyme hydrolysis is time and amount of sulfatase dependent. The rate of hydrolysis is analyte-dependent (MT $>>N M N>M N)$, although it must contain at least $0.8 \mathrm{U} / \mathrm{ml}$ of sample. The Deming regression curves comparing acid versus enzyme hydrolysis on patient samples assessed that both methods gave similar unbiased concentrations.

Conclusion: Enzyme and acid treatments are equivalent and efficient for removing sulfate from metanephrines as long as the optimal protocol is used for each method. However, the gold standard method for acid hydrolysis at 10 min established more than 20 years ago was not satisfactory regarding the hydrolysis of metanephrines in plasma.
\end{abstract}

(C) 2014 Elsevier B.V. All rights reserved.

\section{Introduction}

Biochemical diagnosis of pheochromocytoma relies on the measurement of metanephrines (normetanephrine (NMN), metanephrine $(\mathrm{MN})$ and methoxytyramine (MT)) in urine or/and plasma [1-3]. Metanephrines originate from the methoxylation of catecholamines by catechol-O-methyl-transferase [4]. The monoamine-preferring sulfotransferase SULT1A3 (E.C. number 2.8.2.1) is responsible for the sulfate conjugation of metanephrines, giving rise to hydrosoluble metabolites, considered as end point products eventually eliminated in urines [5]. In the context of pheochromocytoma diagnostic, urine total metanephrines (i.e., free + sulfate conjugated) are currently measured in clinical chemistry laboratories due to high concentration levels (i.e., about 100-1000 nmol/L). In contrast, plasma total metanephrines measurement is less frequently available in laboratories since no commercial kits have been yet developed, necessitating an inhouse analytical validation. The hydrolysis of the sulfate moiety is a

\footnotetext{
* Corresponding author. Tel.: +41 2131404 98; fax: +41 213147835

E-mail address: Eric.Grouzmann@chuv.ch (E. Grouzmann).

1 These authors contributed equally to this work
}

prerequisite to quantify metanephrines since the analytical methodology relies on the redox properties of the free hydroxyl group present on the benzene ring when measuring by electrochemical detection. Besides, no extraction procedures have yet been reported for sulfated metanephrines for LC-MS/MS quantification. We have previously reported that urine sulfated metanephrines are entirely deconjugated by a treatment consisting of boiling acid perchloric for $30 \mathrm{~min}$ at $\mathrm{pH} 1.0$ [6]. Intriguingly, an early study by Pagliari et al. [7] established that by following the same methodology, the hydrolysis time should not last more than $10 \mathrm{~min}$ to prevent loss of metanephrines. Alternatively, several reports demonstrated that sulfated metanephrines may also be efficiently deconjugated with $0.1 \mathrm{U}$ [8] or $0.5 \mathrm{U}$ of sulfatase for $0.2 \mathrm{ml}$ of plasma after $30 \mathrm{~min}$ incubation at $37{ }^{\circ} \mathrm{C}$ [9], or more recently by the same method after $1 \mathrm{~h}$ of incubation with $0.33 \mathrm{U}$ of enzyme [10]. The timedependent discrepancy observed between plasma and urine for acid hydrolysis of sulfated metanephrines prompted us to evaluate whether this phenomenon applies to synthetic sulfated metanephrines at different $\mathrm{pH}$ and time courses. This was aimed to assess the behavior of synthetic compounds when used as an internal control for hydrolysis efficiency. In addition, we studied the hydrolysis rate by sulfatase at different enzyme concentrations and incubation times to establish optimal hydrolysis 
conditions. We then compared enzyme and acid-treated samples to assess whether both methods gave similar results. Finally, patient samples were evaluated by both methodologies to ensure that total metanephrine concentrations reported in publications could be pooled for providing recommendations about normal reference intervals.

\section{Materials and Methods}

\subsection{Reagents, materials and instruments}

The chemical protocols used for the synthesis of each sulfate metanephrines has been previously published [6]. All commercially available reagents and solvents (Fluka/Aldrich, Buchs, $\mathrm{CH}$ and Acros, Wohlen, $\mathrm{CH}$ ) were used without further purification. Sulfatase from Aerobacter aerogenes (S1629), normetanephrine and methoxytyramine were purchased from Sigma-Aldrich (St. Louis, MO, USA), and metanephrine was supplied by Isosciences (King of Prussia, PA, USA). Oasis ${ }^{\circledR}$ WCX $\mu$ Elution Plate $30 \mu \mathrm{m}$ (part no. 186002499) was obtained from Waters (Milford, MA, USA). Matrix used included charcoal-stripped human heparinized plasma (Sera Care Life Sciences, Gaithersburg, MD, USA) and plasma samples from patients.

\subsection{Validation of hydrolysis conditions}

\subsubsection{Acid hydrolysis}

Charcoal-stripped human heparinized plasma samples $(0.2 \mathrm{ml})$ in sextuplicate spiked with $50 \mathrm{nM}$ sulfated normetanephrine (S-NMN), $20 \mathrm{nM}$ sulfated metanephrine (S-MN) and $10 \mathrm{nM}$ sulfated methoxytyramine (S-MT) were thoroughly mixed with $0.040 \mathrm{ml}$ of $2 \mathrm{~mol} / \mathrm{L}$ perchloric acid, vortex mixed for $10 \mathrm{~min}$ on ice to allow protein precipitation and centrifuged for $10 \mathrm{~min}$ at $1800 \mathrm{~g}$. The supernatants $(0.170 \mathrm{~mL})$ were recovered in an Eppendorf tube and mixed with $1 \mathrm{ml}$ of $0.02 \mathrm{~mol} / \mathrm{L}$ perchloric acid. Sulfated metanephrines were hydrolyzed in a boiling bath $\left(100{ }^{\circ} \mathrm{C}\right)$ for $10,20,30,40$ and $60 \mathrm{~min}$, and the hydrolysis process was stopped on ice. Free metanephrines $(0.1 \mathrm{~mL})$ released by hydrolysis were brought to $\mathrm{pH} 6.5$ with $0.1 \mathrm{ml}$ of $25 \mathrm{mM}$ sodium pentaborate containing 1.5 mM EDTA. The samples were then purified by solid phase extraction and quantified by tandem mass spectrometry [11].

\subsubsection{Enzyme hydrolysis}

Charcoal-stripped human heparinized plasma samples $(0.2 \mathrm{ml})$ in sextuplicate spiked with $50 \mathrm{nM}$ S-NMN, $20 \mathrm{nM}$ S-MN and $10 \mathrm{nM} \mathrm{S-MT}$ were mixed with $0,0.005,0.01,0.02,0.04,0.08,0.16$ and $0.33 \mathrm{U}$ of sulfatase to evaluate the efficiency of hydrolysis. The mixture incubation was performed during 15,30 and $60 \mathrm{~min}$ at $37^{\circ} \mathrm{C}$ under gentle shaking. Free metanephrines produced after the enzyme hydrolysis process were purified by solid phase extraction and quantified by tandem mass spectrometry [11].

\subsubsection{Hydrolysis method comparison}

Plasma total metanephrine concentrations in plasma samples collected from 62 patients screened for pheochromocytoma were determined by HPLC with electrochemical detection (Coularray system; ESA-Dionex, Sunnyvale, CA USA) as routinely performed in our laboratory [1]. Results obtained after $10 \mathrm{~min}$ and $30 \mathrm{~min}$ of acid hydrolysis were compared with those observed after $30 \mathrm{~min}$ of enzyme hydrolysis.

\subsection{Statistical analyses}

Results were reported as dot plots after ANOVAs and, when necessary, post hoc Scheffe analyses. Deming regression curves and BlandAltman plots were statistically analyzed using the Analyse-it (version 2) add-on package for Microsoft Excel.

\section{Results and discussion}

\subsection{Acid hydrolysis}

We observed that synthetic sulfated metanephrines are not spontaneously hydrolyzed in plasma in acidic conditions as long as they are not heated at $100{ }^{\circ} \mathrm{C}$ (Fig. 1). Only $60 \%$ of sulfated metanephrines are hydrolyzed after $10 \mathrm{~min}$ of incubation in boiling water (Fig. 1). Rising the acid
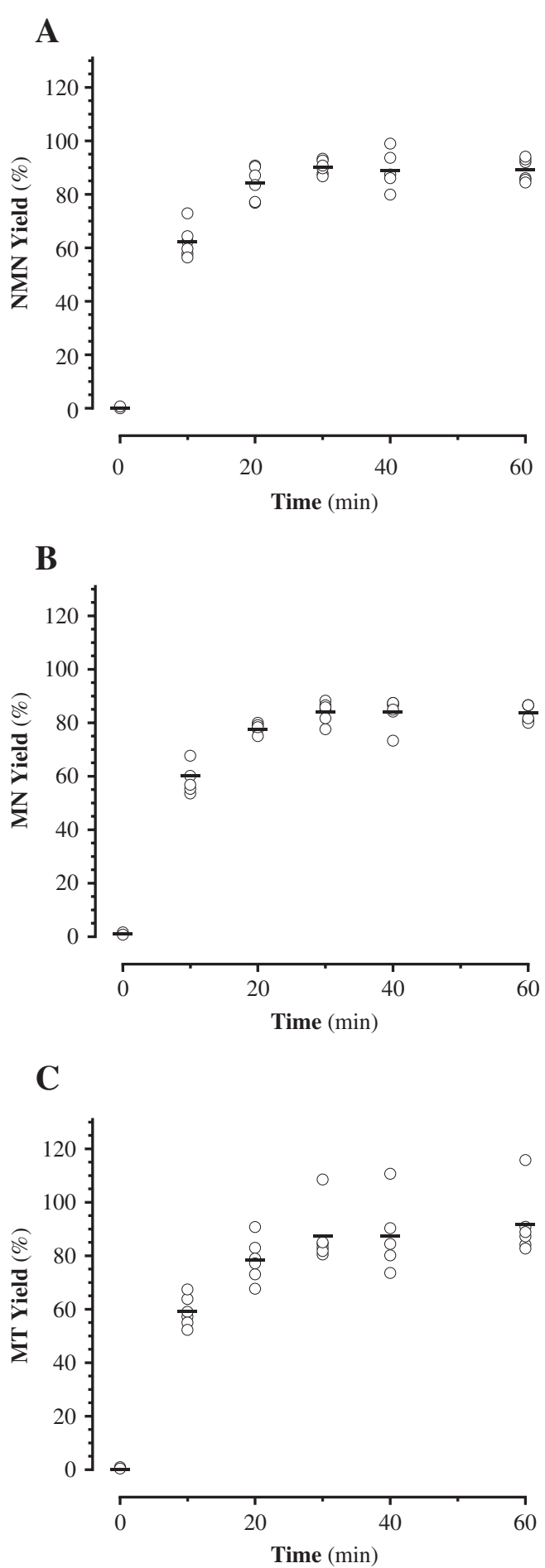

Fig. 1. Quantification of NMN (A), MN (B) and MT (C) in charcoal-stripped plasma complemented with S-MNs $50 \mathrm{nM}$ sulfated normetanephrine (S-NMN), 20 nM sulfated metanephrine (S-MN) and $10 \mathrm{nM}$ sulfated methoxytyramine (S-MT) heated over a boiling bath $\left(100{ }^{\circ} \mathrm{C}\right)$ for $10,20,30,40$ and $60 \min (n=6)$. Values were expressed in \% of MNs concentrations hydrolyzed. Mean values are indicated by a line for each sextuplicate. 
hydrolysis time significantly raised the yield of deconjugation after 20 min ( $p<0.001$ for NMN-S and MN-S and $p<0.05$ for MT-S, compared to $10 \mathrm{~min}$ ) with a plateau after $30 \mathrm{~min}$ of incubation. Further hydrolysis up to $60 \mathrm{~min}$ incubation did not improve the hydrolysis yield (yield ranged between $84 \%$ and $92 \%$ ). The data for hydrolysis of NMN-S and MN-S were normally distributed accordingly to the Shapiro-Wilk test, whereas MT-S data were not normally distributed probably reflecting a higher variability for the deconjugation of this analyte. These results are in agreement with our previous work on urines [6], indicating that $30 \mathrm{~min}$ is ideal to hydrolyze sulfated metanephrines and that the nature of the matrix does not affect the hydrolysis rate as long as the $\mathrm{pH}$ is close to 1.0 ( $\mathrm{pH}$ at 1.2 in our condition). However, our data are in total discrepancy with the work of Pagliary et al. [7], who found a maximum concentration of the deconjugation of plasma S-NMN and S-MN submitted to acid hydrolysis after only $10 \mathrm{~min}$ and gradually lower concentrations of deconjugated metanephrines when incubation time was 20 and $30 \mathrm{~min}$.

\subsection{Enzyme hydrolysis}

Enzyme hydrolysis is time- and amount of enzyme-dependent. Exposure to $0.33 \mathrm{U}$ of sulfatase during $15 \mathrm{~min}$ at $37{ }^{\circ} \mathrm{C}$ resulted in the $100 \%$ deconjugation of all sulfated metanephrines (Fig. 2). The yield of hydrolysis is dependent on the analyte involved with an order of sensitivity for hydrolysis S-MT > > S-NMN $>$ S-MN. The hydrolysis of the three sulfate metanephrines was not normally distributed due to a significant variability for the deconjugation especially at low enzyme concentrations. Consequently, $15 \mathrm{~min}$ of incubation with $0.05 \mathrm{U}$ of sulfatase is required to fully remove sulfate from S-MT (Fig. 2C), $0.16 \mathrm{U}$ for S-NMN (Fig. 2A) and $0.33 \mathrm{U}$ for S-MN (Fig. 2B). Our data indicated that $0.16 \mathrm{U}$ of enzyme added for $30 \mathrm{~min}$ to $0.2 \mathrm{~mL}$ plasma is the optimal way to achieve the full hydrolysis of the sulfate moiety for the three sulfated metanephrines. Our finding is intermediate between similar methods using $0.1 \mathrm{U}$ [8] or $0.5 \mathrm{U}$ of sulfatase for $0.2 \mathrm{ml}$ of plasma during 30 min incubation at $37{ }^{\circ} \mathrm{C}$ [9] and certainly spares more time than the previously proposed $1 \mathrm{~h}$ incubation with $0.33 \mathrm{U}$ of enzyme [10].

\subsection{Hydrolysis method comparison}

Deming regression curves performed in plasma samples collected from 62 patients confirmed the results observed with synthetic sulfated metanephrines. We observed an underestimation of about $40 \%$ of the concentrations of S-NMN, S-MN and S-MT in acid-deconjugated samples for $10 \mathrm{~min}$ compared to $30 \mathrm{~min}(p<0.0001)$. In contrast, the concentrations of total metanephrines in samples treated with $0.16 \mathrm{U}$ sulfatase or acid for $30 \mathrm{~min}$ gave similar results, and no systematic or proportional bias was observed (see Figs. S1A-S1D, Supplementary data). Bland-Altman plots for the mean difference between the 62 sample treatments illustrated the differences found in the Deming regression curves (see Fig. S2A-S2D, Supplementary data).

Our laboratory has established local reference ranges for plasma total metanephrines measured for the diagnosis of pheochromocytoma in patients investigated in clinical wards in Switzerland [1]. Since plasma total metanephrines are determined after a deconjugation step including a 10-min acid hydrolysis according to the protocol established by Pagliari et al. [7], we expected a 30\%-40\% underestimation of real concentrations of sulfated metanephrines. Our upper reference limits based on a 97.5 percentile for total metanephrines in hypertensive patients referred for biochemical measurements because of the presence of clinical signs suggestive of a pheochromocytoma that was finally excluded is $29.92 \mathrm{nmol} / \mathrm{L}$ for NMN and $11.26 \mathrm{nmol} / \mathrm{L}$ for MN and $9.31 \mathrm{nmol} / \mathrm{L}$ for NMN and $5.35 \mathrm{nmol} / \mathrm{L}$ for $\mathrm{MN}$ in healthy subjects [1]. These concentrations are similar to those reported by D'herbonez et al. [12] that used the same protocol that we used to deconjugate metanephrines. Since adequate sulfatase treatment should increase these concentrations by $30 \%-40 \%$, we would expect
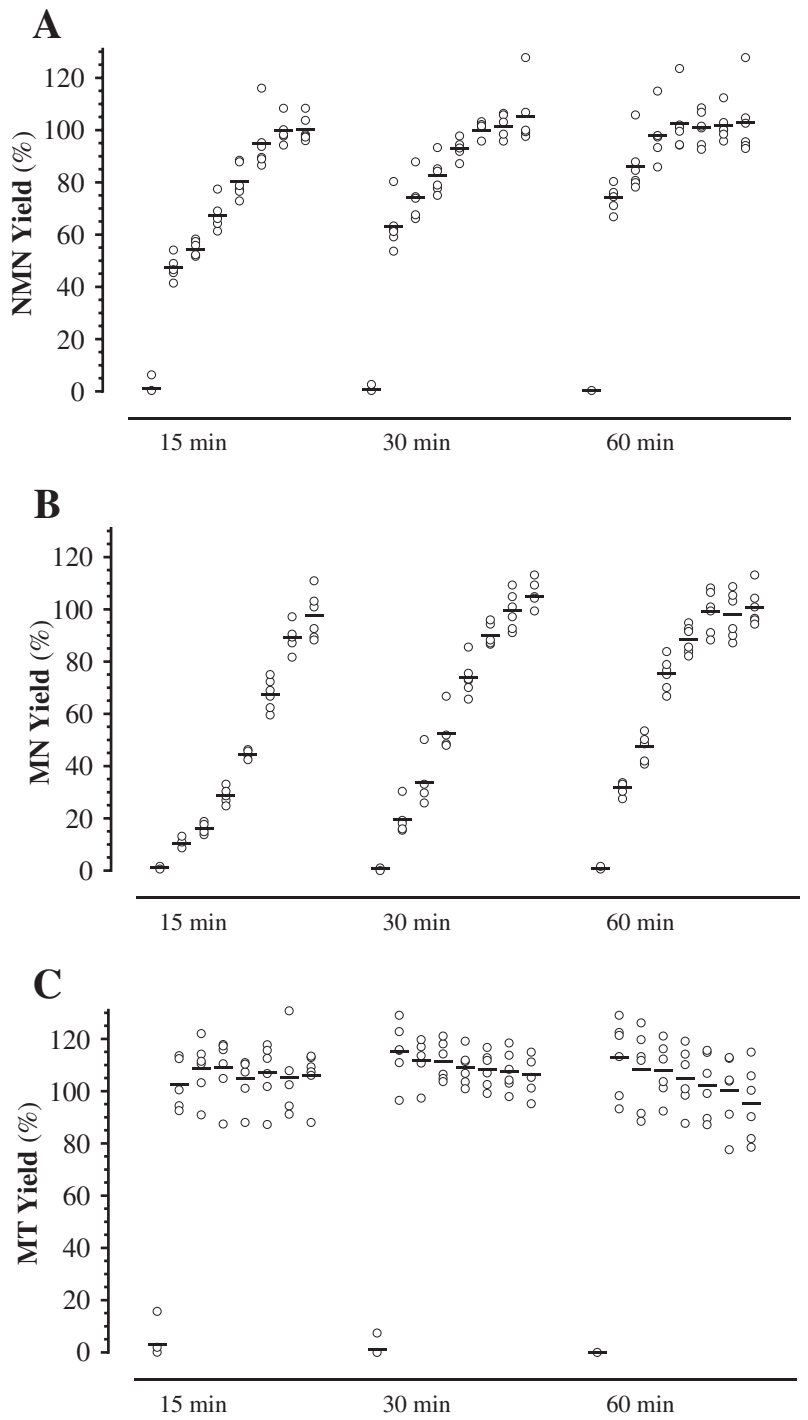

Fig. 2. Quantification of NMN (A), MN (B) and MT (C) in charcoal-stripped plasma complemented with S-MNs in presence of $0,0.005,0.01,0.02,0.04,0.08,0.16$ and 0.33 $\mathrm{U}$ of sulfatase (increasing concentrations from the left to the right column). The mixture incubation was performed during 15,30 and $60 \mathrm{~min}$ at $37^{\circ} \mathrm{C}(n=6)$. Values were expressed in \% of MNs concentrations hydrolyzed. Mean values are indicated by a line for each sextuplicate.

that these reference ranges should be reset to $40 \mathrm{nmol} / \mathrm{L}$ for NMN and $15 \mathrm{nmol} / \mathrm{l}$ for MN. Pamporaki et al. [9] also reported reference intervals for total metanephrine measurements based on sulfatase deconjugation that included a mix of healthy volunteers and patients with primary hypertension and reported similar values at $25.4 \mathrm{nmol} / \mathrm{L}$ for NMN and $9.2 \mathrm{nmol} / \mathrm{L}$ for MN (incubation with $0.5 \mathrm{U} / 0.2 \mathrm{ml}$ plasma of sulfatase for $30 \mathrm{~min}$ ), but this population is not similar to ours and is not truly representative of patients for which a pheochromocytoma is suspected; instead, the patients for which a pheochromocytoma is excluded are more representative to establish a cutoff, but unfortunately, these values are not provided in this article [9]. Eisenhofer et al. [10] previously reported that incubation of plasma with $0.33 \mathrm{U}$ of sulfatase for $1 \mathrm{~h}$ was enough to produce unconjugated metanephrines. They reported a 97.5 percentile at $31 \mathrm{nmol} / \mathrm{l}$ for NMN and $10.3 \mathrm{nmol} / \mathrm{L}$ for MN for patients with primary hypertension. These results were close to our data published with 10-min acid hydrolysis. A previous study from the same group, however, established a lower upper reference limit at $16.5 \mathrm{nmol} / \mathrm{L}$ for NMN and similar concentrations for $\mathrm{MN}$ at $10.9 \mathrm{nmol} / \mathrm{L}$ [8] for hypertensive patients. This discrepancy for only NMN is surprising since a lower amount of sulfatase $(0.1 \mathrm{U} / 0.2 \mathrm{ml}$ of plasma for $30 \mathrm{~min})$ 
was expected to preferentially affect MN deconjugation since S-MN proves to be less prone to desulfonation than both S-MT and S-NMN.

All these differences are most likely due to differences in how the hypertensive control group was defined and taking or not into account the effect of the combination of anti-hypertensive treatments that may affect the sympathetic nervous activity and formal evaluation of renal failure for these patients since sulfated metanephrines are eliminated through the kidney [10].

In conclusion, we have established the experimental conditions that allow for a full deconjugation of sulfated metanephrines. Acid or enzyme ( $0.16 \mathrm{U} / 0.2 \mathrm{ml}$ plasma) hydrolysis for $30 \mathrm{~min}$ resulted in similar efficiency. Sulfatase treatment costs may be higher than the acid hydrolysis step, but it is compensated by a significant gain in turnaround time treatment since neither precipitation step of plasma proteins nor $\mathrm{pH}$ adjustment prior to SPE are mandatory. The use of synthetic sulfated metanephrines is also a prerequisite to ensure successful MN deconjugation. Finally, this study also highlights the need to establish interval references for sulfated metanephrines based on a common definition of the referral population to be compared with patients having a pheochromocytoma (hypertensive patients with signs suggestive of a pheochromocytoma, hypertensive patients resistant to anti-hypertensive treatment, patients presenting an incidentaloma, patients previously having a pheochromocytoma and monitored for possible relapse and healthy subjects bearing a mutation for a gene predisposing for a pheochromocytoma).

\section{Acknowledgements}

The Swiss National Science Foundation and the EPFL are gratefully acknowledged for financial support (SGL). We thank Dr. Thierry Buclin for statistical assistance.

\section{Appendix A. Supplementary data}

Supplementary data to this article can be found online at http://dx. doi.org/10.1016/j.cca.2013.12.044.

\section{References}

[1] Grouzmann E, Drouard-Troalen L, Baudin E, et al. Diagnostic accuracy of free and tota metanephrines in plasma and fractionated metanephrines in urine of patients with pheochromocytoma. Eur J Endocrinol 2010;162:951-60.

[2] Sawka AM, Jaeschke R, Singh RJ, Young WF. A comparison of biochemical tests for pheochromocytoma: measurement of fractionated plasma metanephrines compared to the combination of 24-hour urinary metanephrines and catecholamines. J Clin Endocrinol Metab 2003;88:553-8.

[3] Lenders JW, Pacak K, Walther MM, et al. Biochemical diagnosis of pheochromocytoma: which test is best? JAMA 2002;287:1427-34.

[4] Goldstein DS, Eisenhofer G, Kopin IJ. Sources and significance of plasma levels of catechols and their metabolites in humans. J Pharmacol Exp Ther 2003;305:800-11.

[5] Falany CN. Enzymology of human cytosolic sulfotransferases. FASEB J 1997;11:206-16

[6] Simonin J, Gerber-Lemaire S, Centeno C, et al. Synthetic calibrators for the analysis of total metanephrines in urine: revisiting the conditions of hydrolysis. Clin Chim Acta 2012;413(11-12):998-1003.

[7] Pagliari R, Cottet-Emard JM, Peyrin L. Determination of free and conjugated normetanephrine and metanephrine in human plasma by high-performance liquid chromatography with electrochemical detection. J Chromatogr 1991;563:23-36.

[8] Lenders JW, Keiser HR, Goldstein DS. Plasma metanephrines in the diagnosis of pheochromocytoma. Ann Intern Med 1995;123:101-9.

[9] Pamporaki C, Därr R, Bursztyn M, et al. Plasma-free vs deconjugated metanephrines for diagnosis of phaeochromocytoma. Clin Endocrinol (Oxf) 2013. http://dx.doi.org/ 10.1111/cen.12191.

[10] Eisenhofer G, Huysmans F, Pacak K, et al. Plasma metanephrines in renal failure. Kidney Int 2005;67:668-77.

[11] Grouzmann E, Matter M, Bilz S, et al. Monoamine oxidase A down-regulation contributes to high metanephrine concentration in pheochromocytoma. J Clin Endocrinol Metab 2012;97:2773-81.

[12] d'Herbomez M, Forzy G, Bauters C, et al. An analysis of the biochemical diagnosis of 66 pheochromocytomas. Eur J Endocrinol 2007;156:569-75. 